\title{
Brechas de género en competencias cognitivas: evidencia internacional
}

\author{
Sara de la Rica \\ Universidad del País Vasco e ISEAK* \\ Yolanda F. Rebollo-Sanz \\ Universidad Pablo de Olavide
}

\section{Resumen}

Este trabajo documenta brechas de género en competencias cognitivas, como comprensión lectora y competencias matemáticas, para una muestra conjunta de 31 países de la OCDE. Se hace uso del Programa para la Evaluación de Competencias de Adultos auspiciado por la OCDE (PIAAC 2012). Se encuentra una brecha de género entre el 4 y el 5 por 100 a favor de los varones. Al ajustar por diferencias en capital humano y en condiciones laborales, la brecha decrece solo marginalmente. Un análisis exploratorio posterior nos indica que aspectos intangibles como la ansiedad/tensión frente a las matemáticas detectada ya a la edad de los 14 años puede estar detrás de la existencia de estas brechas de género en los adultos.

Palabras clave: competencias cognitivas, brechas de género, PIAAC, brechas ajustadas, intangibles.

Clasificación JEL: J31, J71.

\begin{abstract}
This paper documents gender gaps in cognitive skills, such as reading comprehension and mathematical skills, for a joint sample of 31 OECD countries. The Adult Competency Assessment Program sponsored by the OECD is used (PIAAC 2012). There is a gender gap in math skills between 4 and 5 per cent in favor of males. When adjusting for differences in human capital and working conditions, the gap decreases only marginally. A subsequent exploratory analysis indicates that intangible aspects such as anxiety / tension versus mathematics detected at the age of 14 may be behind the existence of these gender gaps in adults.
\end{abstract}

Keywords: cognitive competences, gender gaps, PIAAC, adjusted gender gaps, intangibles.

JEL classification: J31, J71.

\section{Introducción}

El capital humano es un input fundamental en la denominada sociedad del conocimiento, nuestra sociedad actual. Tradicionalmente, el concepto del capital humano y su medición se han remitido a la productividad del trabajador, la cual se ha relacio-

\footnotetext{
* Initiative for socio-economic analysis and knowledge.
} 
nado fundamentalmente con su nivel formativo. Sin embargo, la literatura empírica más reciente apunta que esta variable es un indicador impreciso del nivel de capital humano, al mismo tiempo que plantea la necesidad de buscar indicadores más adecuados que se aproximen mejor al concepto de habilidad/competencias del individuo (OECD, 2012; Hanushek, 2015). Este límite del nivel formativo como indicador aproximado del capital humano es especialmente relevante cuando el objetivo es evaluar y entender el comportamiento y los determinantes de las brechas de género en el mercado de trabajo. En los últimos veinte años ha habido un intenso proceso de convergencia en los niveles formativos entre los hombres y las mujeres, y de hecho, hoy en día, en muchos países el porcentaje de mujeres con estudios universitarios, así como los resultados obtenidos por estas, son superiores a los de los hombres (OCDE, 2018). Sin embargo, la brecha de género en diferentes ámbitos del mercado laboral (es decir, salarios, porcentaje de contratos a tiempo completo, etc.) no solo sigue existiendo, sino que parece haber dejado de reducirse. En consecuencia, a pesar de los logros conseguidos en estos últimos años, es preciso seguir analizando los determinantes de la brecha de género no solo porque esta es intrínsecamente injusta, sino también porque numerosos estudios sugieren que una mayor igualdad de género conduce a un mejor desempeño económico ${ }^{1}$. Esto motiva la búsqueda de explicaciones alternativas para entender los determinantes de la brecha de género en el mercado de trabajo (Olivetti y Petrongolo, 2016) y diseñar así las políticas adecuadas que favorezcan su desaparición. Dentro de esta línea de investigación podemos destacar dos enfoques complementarios predominantes: por un lado, identificar mejores indicadores de las habilidades del individuo y por otro, relacionar las brechas de género con normas culturales y sociales que a su vez influyen en las habilidades futuras de los individuos (Blau y Khan, 2017).

En esta última línea de estudio empírico se enmarca el presente trabajo. En concreto, analizamos si existen brechas de género en las habilidades cognitivas relacionadas con las habilidades numérica y de lectura. Para ello nos beneficiamos de la información que proporciona el Programa para la Evaluación de Competencias de Adultos auspiciado por la OCDE (The Programme for the International Assessment of Adult Competencies, PIAAC). Este programa mide habilidades cognitivas y laborales clave, con el objeto de aproximar las destrezas básicas que permiten a las personas adultas participar en la vida social y económica del siglo xxI, así como dotarles de competencias laborales muy necesarias y valoradas en el trabajo del futuro. En particular, en este trabajo se consideran las competencias cognitivas que determinan la capacidad de cálculo y la comprensión lectora. Adicionalmente, la encuesta recopila información muy interesante sobre los antecedentes personales, sobre el hogar, además de información sobre el tipo y las exigencias competenciales del puesto de

\footnotetext{
${ }^{1}$ Según datos del Foro Económico Mundial (2012), la paridad económica de género podría agregar 250.000 millones de dólares adicionales al PIB del Reino Unido y 1.750 .000 millones de dólares al de Estados Unidos. Con la tasa de progreso actual, la brecha global de género global tardará cien años en cerrarse, mientras que la brecha en el lugar de trabajo no se cerrará en 217 años. Es una brecha que el mundo no puede permitirse ignorar.
} 
trabajo. La prueba se aplica a personas entre 16 y 65 años en sus hogares en 31 países de la OCDE ${ }^{2}$.

Varios estudios empíricos han demostrado que las habilidades cognitivas están altamente correlacionadas con la probabilidad de éxito en el mercado de trabajo (es decir, salarios altos y estabilidad laboral); es más, se ha encontrado un efecto causal entre las habilidades cognitivas relacionadas con las matemáticas y los altos salarios (Hanuseck et al., 2014; Hanushek y Woessmann, 2012; Oreopoulos y Salvanes, 2011; Riddell y Song, 2011). Básicamente, mejores habilidades cognitivas se traducen en una mejor comprensión del entorno y una mejor ejecución de las tareas. Este efecto positivo de las habilidades cognitivas en la productividad del individuo se va retroalimentando en el tiempo, favoreciendo mejores empleos con salarios más altos (Hanushek y Woessmann, 2012). Por tanto, la existencia de brechas de género en las habilidades cognitivas puede ser un factor explicativo de las brechas de género en participación laboral y en salarios que aún hoy existen en muchas economías desarrolladas.

En el presente trabajo se trata de aprovechar esta novedosa fuente de información de habilidades cognitivas para un amplio conjunto de países con el objeto de estudiar si existen brechas de género en dichas competencias y si existen, evaluar sus determinantes fundamentales.

Encontramos que no existen brechas de género en términos de competencia lectora pero sí existen brechas de género en las competencias matemáticas. Dichas brechas de género son comunes a casi todos los países considerados en la muestra y para diferentes tipos de individuo, ya sean agrupados por niveles de edad, por niveles y ramas de estudio o por tipo de ocupación. También encontramos pequeñas diferencias entre las brechas de género sin ajustar y las brechas de género ajustadas por características individuales y laborales en la habilidad numérica, lo que apunta a que otros factores culturales o sociales deben estar detrás de las brechas de género encontradas en matemáticas. En la última sección hacemos una incursión explorativa sobre estos posibles factores.

El presente artículo se estructura como sigue. En la sección dos se incluye una detallada estadística descriptiva de las habilidades cognitivas consideradas en el trabajo. En la sección tres presentamos la estrategia empírica a seguir y los principales resultados de los modelos estimados. Finalmente presentamos una sección de resumen y principales conclusiones.

${ }^{2}$ La OCDE recoge la información de esta encuesta entre agosto de 2011 y marzo de 2012. Los países participantes en PIAAC son los siguientes: Austria, Bélgica, Canadá, República Checa, Alemania, Dinamarca, España, Estonia, Finlandia, Francia, Gran Bretaña, Irlanda, Italia, Japón, Corea del Sur, Holanda, Noruega, Polonia, Rusia, Eslovaquia, Suecia y los Estados Unidos. La base de datos se compone de 166.000 observaciones. 


\section{Brechas de género en competencias cognitivas: evidencia descriptiva}

Comenzamos presentando un análisis incondicional de las brechas de género en las habilidades cognitivas relativas a lectura y matemáticas.

En todos los cuadros que se presentan a continuación se incluye la puntuación media y la desviación estándar (d.e.) ${ }^{3}$ de los test de competencias en matemáticas y en comprensión lectora por género para dos colectivos, a saber, para toda la muestra (población trabajadora, parada e inactiva) y para el colectivo de personas con mayor relación con el mercado laboral, bien por estar ocupados, o parados, pero con una experiencia de empleo reciente. A este último colectivo se le denominará a partir de ahora el colectivo de personas ocupadas. Se presenta la puntuación para ambos colectivos para poder tener en cuenta posibles sesgos en la selección de participar en el mercado laboral que pudiesen dar lugar a diferencias en las brechas de género en estas competencias ${ }^{4}$. La evaluación de dichas habilidades cognitivas se presenta en una escala de 1 a 500 puntos, siguiendo las directrices de la propia encuesta.

\subsection{Brechas de género en competencias cognitivas: totales y por grupos de edad}

El primer aspecto a destacar del Cuadro 1 se refiere a la notable brecha de género que se observa en matemáticas ${ }^{5}$. Si atendemos en primer lugar al colectivo de todos los trabajadores, independientemente de que estén o no ocupados (primer panel), los datos revelan una desventaja media de las mujeres frente a los varones del 4,88 por 100 que solo se reduce al 4,0 por 100 para el colectivo de trabajadores.

Si además fijamos nuestra atención en las diferencias por edad, diríamos que en general las diferencias de género crecen con la edad, aunque no de una manera espectacular. Si en lugar de observar el colectivo total restringimos el análisis al colectivo de ocupados, se observan algunos aspectos diferenciales: en primer lugar, la brecha de género es prácticamente nula para el tramo más joven. Al igual que lo observado al considerar a todos los trabajadores, la brecha crece con la edad, pero es sensiblemente menor en cualquier tramo de edad a la observada para toda la muestra. Estas diferencias revelan que las mujeres ocupadas no son una muestra representativa de todas las mujeres en cuanto a competencias en matemáticas se refiere, sino que en media tienen mayores competencias cognitivas. También se observa que los

3 Todos los estadísticos de la sección se computan teniendo en cuenta los pesos muestrales de PIAAC.

${ }^{4}$ En PIAAC, cada individuo responde solo a un subconjunto de las preguntas que forman parte de la evaluación de las competencias lectoras y numéricas y se usan técnicas de imputación que permiten obtener un indicador completo de dichas competencias para cada individuo. Nuestro análisis tiene en cuenta estas características especiales de la base de datos. En la estadística descriptiva y en el análisis econométrico usamos la media del conjunto de valores posibles -diez para cada habilidad- como variable a explicar. Esta media es un indicador consistente y permite ofrecer los resultados de la estimación de forma más concisa.

5 Se ha considerado como primer intervalo de edad a las personas entre 24 y 29 años para captar las brechas desde el inicio de la etapa laboral. Por debajo de 24 años, la población activa es muy escasa en la muestra. 


\section{CUADRO 1}

\section{HABILIDADES COGNITIVAS EN COMPETENCIA LECTORA Y MATEMÁTICAS TOTALES Y POR EDAD}

\begin{tabular}{|c|c|c|c|c|c|c|c|}
\hline & \multirow{2}{*}{ Edad } & \multicolumn{2}{|c|}{ Mujeres } & \multicolumn{2}{|c|}{ Hombres } & \multirow{2}{*}{$\begin{array}{c}\text { Diferencia } \\
(\%)\end{array}$} \\
\hline & & & Media & d.e. & Media & d.e. & \\
\hline \multirow{8}{*}{$\begin{array}{c}\text { Todos los } \\
\text { individuos }\end{array}$} & Matemáticas & $24-29$ & 263 & 48 & 276 & 49 & $-2,49$ \\
\hline & & $30-39$ & 263 & 51 & 278 & 50 & $-4,92$ \\
\hline & & $40-49$ & 256 & 51 & 269 & 53 & $-5,66$ \\
\hline & & Total & 259 & 49 & 272 & 51 & $-4,88$ \\
\hline & Lectura & $24-29$ & 281 & 43 & 278 & 48 & 0,17 \\
\hline & & $30-39$ & 276 & 45 & 281 & 48 & $-0,29$ \\
\hline & & $40-49$ & 270 & 45 & 275 & 51 & $-1,26$ \\
\hline & & Total & 274 & 44 & 276 & 46 & $-0,26$ \\
\hline \multirow[t]{8}{*}{ Trabajadores } & Matemáticas & $24-29$ & 271 & 45 & 278 & 48 & $-0,13$ \\
\hline & & $30-39$ & 272 & 46 & 281 & 48 & $-2,60$ \\
\hline & & $40-49$ & 264 & 46 & 275 & 51 & $-3,24$ \\
\hline & & Total & 268 & 46 & 276 & 49 & $-4,00$ \\
\hline & Lectura & $24-29$ & 287 & 40 & 282 & 43 & 2,90 \\
\hline & & $30-39$ & 284 & 41 & 283 & 44 & 1,64 \\
\hline & & $40-49$ & 276 & 42 & 276 & 45 & 0,57 \\
\hline & & Total & 281 & 41 & 279 & 45 & 0,14 \\
\hline
\end{tabular}

FUENTE: PIAAC.

NOTA: Los valores medios de cada habilidad oscilan entre 1 y 500 puntos, siguiendo las directrices de la propia encuesta PIAAC.

varones ocupados presentan en media, mejores competencias en matemáticas relativamente al total de varones, pero la diferencias entre los varones ocupados y el total de varones es sensiblemente menor a las observadas entre las mujeres ocupadas y el total de mujeres. Esta mayor diferencia en las mujeres es precisamente la que provoca que la brecha de género en competencias en matemáticas sea menor en el colectivo de trabajadores que al considerar a todos los individuos.

Un aspecto interesante a entender es la razón o razones por las cuales las brechas de género en competencias cognitivas en el área de las matemáticas crecen más contundentemente con la edad para el total de individuos, mientras que para el colectivo de personas ocupadas solo surge a partir de los 30 años y crece con la edad de forma menos pronunciada. Dado que tenemos una sección cruzada de datos, no es posible discernir entre un efecto edad (la brecha en competencias crece a medida que se envejece) o un efecto cohorte (las personas que tienen diferentes edades en la muestra pertenecen a cohortes diferentes). Existe evidencia empírica reciente (Kleven et al., 2019) que confirma que a partir de la entrada en la maternidad, las trayectorias laborales de las mujeres divergen sensiblemente de sus homólogos varones. Si ese fuera el caso, esta divergencia podría resultar en una menor competencia en matemáticas de las mujeres en comparación con los varones, lo que explicaría el aumento más 
pronunciado de la brecha a partir de los treinta años que se documenta en el Cuadro 1. En secciones posteriores trataremos de profundizar en estos factores para ver si es posible entender el mecanismo que provoca esta divergencia en competencias matemáticas de hombres y mujeres con la edad.

Con respecto a la competencia lectora, el Cuadro 1 destaca que las diferencias de género son prácticamente nulas, tanto en media como para cualquier intervalo de edad, e independientemente de que las personas trabajen o no.

\subsection{Brechas de género en competencias cognitivas: por niveles educativos y áreas de estudio}

Una segunda pregunta relevante a abordar es si las brechas de género en las competencias cognitivas varían o no en función del nivel educativo alcanzado, en primer lugar, así como en función del área de estudio seleccionada, en segundo. Es cierto que en las últimas décadas estamos asistiendo, en la mayoría de los países desarrollados, a una masiva entrada de mujeres en educación superior, mayormente universitaria. Este fenómeno ha contribuido positivamente a la reducción de las brechas de género en tasas de participación laboral y salarios. Sin embargo, no es menos cierto que las áreas de estudio fundamentalmente escogidas por las mujeres son las áreas de salud, educación y humanidades. La presencia femenina en áreas STEM (Science, Technology, Economics and Maths) es minoritaria en la mayoría de los países, y de hecho, el objetivo de incrementar la presencia de mujeres en estudios superiores de áreas STEM está hoy en la agenda de la mayoría de las instituciones nacionales e internacionales de los países más desarrollados. Es precisamente esta área de estudio la que exige mayores competencias en matemáticas y la que está más estrechamente correlacionada con trayectorias laborales estables y en empleos altamente remunerados. De ahí la importancia de conocer posibles disparidades en la brecha de género en competencias matemáticas según el área de estudio elegida. Esto nos permitirá entender si las brechas de género mostradas en la sección anterior se producen fundamentalmente entre áreas de estudio diferentes o dentro de las mismas áreas de estudio.

El Cuadro 2 presenta la evidencia de las brechas de género en competencias matemáticas por nivel educativo para toda la población y para los empleados. Se observa que la desventaja de las mujeres frente a los hombres es especialmente aguda entre personas con niveles educativos muy bajos (primaria o menos), superando el 9 por 100. Sin embargo, dicha desventaja de las mujeres no muestra una tendencia decreciente con respecto al nivel educativo. De hecho, la desventaja menor de la mujer se produce cuando se comparan hombres y mujeres solo con educación secundaria, desventaja que alcanza el 3,9 por 100. Para aquellos hombres y mujeres con estudios más allá de secundarios e incluso con estudios universitarios, la desventaja de las mujeres vuelve a crecer hasta situarse alrededor del 5,5 por 100. Con respecto a las brechas de género en competencia lectora, se observa una desventaja de las mujeres frente a los hombres tanto entre colectivos con niveles educativos muy bajos como 


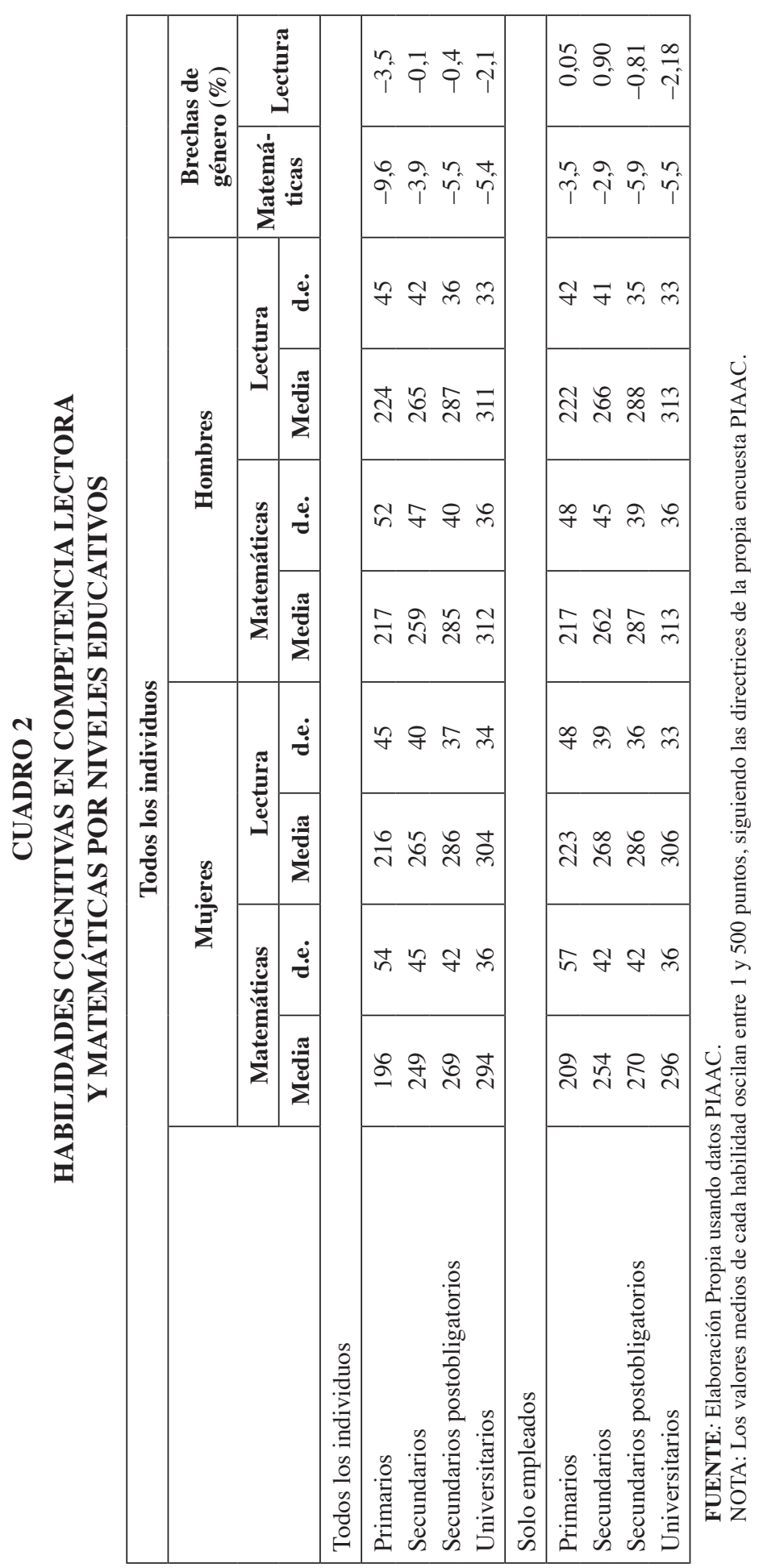


entre quienes tienen estudios superiores. La brecha es, en cualquier caso, pequeña, menos de la mitad de la encontrada para las competencias en matemáticas. Cuando restringimos el análisis a la población empleada, es interesante observar que las brechas de género en matemáticas se reducen notablemente entre los individuos con estudios primarios y secundarios. Por el contrario, son bastante similares para los subgrupos de individuos con niveles de estudios superiores.

Centrándonos en el área de estudio, que precisa mejor el tipo de formación adquirido más allá del nivel educativo, el Cuadro 3 presenta las brechas de género por área de estudio para todos los individuos, así como para diversos colectivos (universitarios, ocupados). Se incluye, además, el porcentaje de mujeres por áreas de estudio, lo que permite identificar cuáles son las áreas de estudios más o menos feminizadas.

En primer lugar, es destacable que, absolutamente en todas las áreas, tengan un mayor o menor porcentaje de mujeres, las brechas de género son notables, y exceptuando el área de humanidades, oscilan entre el 3 y el 5,5 por 100. Este dato es cuanto menos sorprendente, pues hubiéramos esperado encontrar menor disparidad de género entre hombres y mujeres con preferencias similares en cuanto al área de matemáticas o similares. En la segunda parte del Cuadro 3 se restringe la muestra al colectivo de personas con formación universitaria (presumiblemente más homogéneo). Como era de esperar, las brechas de género en matemáticas se reducen en todas las áreas de estudio, pero salvo en el área de estudios generales o agricultura, en el resto, estas brechas oscilan entre el 3,5 y el 5 por 100. Por tanto, está claro que la formación y el área de estudio son elementos fundamentales para entender las brechas de género en competencias analíticas, pero estas brechas no desaparecen cuando comparamos individuos similares en ambas dimensiones. Estos resultados se mantienen para la muestra de empleados -tercer bloque del Cuadro 3.

En segundo lugar, se observa que hay áreas de estudio claramente feminizadas que coinciden con áreas donde la competencia en matemáticas alcanza valores inferiores a la media, como son Humanidades y Salud y Bienestar Social. Esta segunda evidencia es también importante, ya que el área de estudio es una decisión del individuo y habría que entender los factores que motivan las diferencias en las áreas de estudio por género, puesto que influyen directamente en las competencias adquiridas en matemáticas.

\subsection{Brechas de género en competencias cognitivas según la ocupación}

En los estudios de brechas de género se suele destacar la importancia que tiene la segregación ocupacional para entender el comportamiento de la brecha salarial. Los hombres y las mujeres ocupan empleos diferentes y esta segregación ocupacional explica parte de la brecha salarial encontrada. Es pertinente, por tanto, documentar, similarmente a como hemos hecho anteriormente al medir las brechas en competencias dentro de áreas de estudio específicas, si existe segregación ocupacional y si existen disparidades en las diferencias de género dentro de cada ocupación. El Cuadro 4 presenta dicha evidencia descriptiva. Se han desagregado las ocupaciones a un dígito (nueve ocupaciones). 


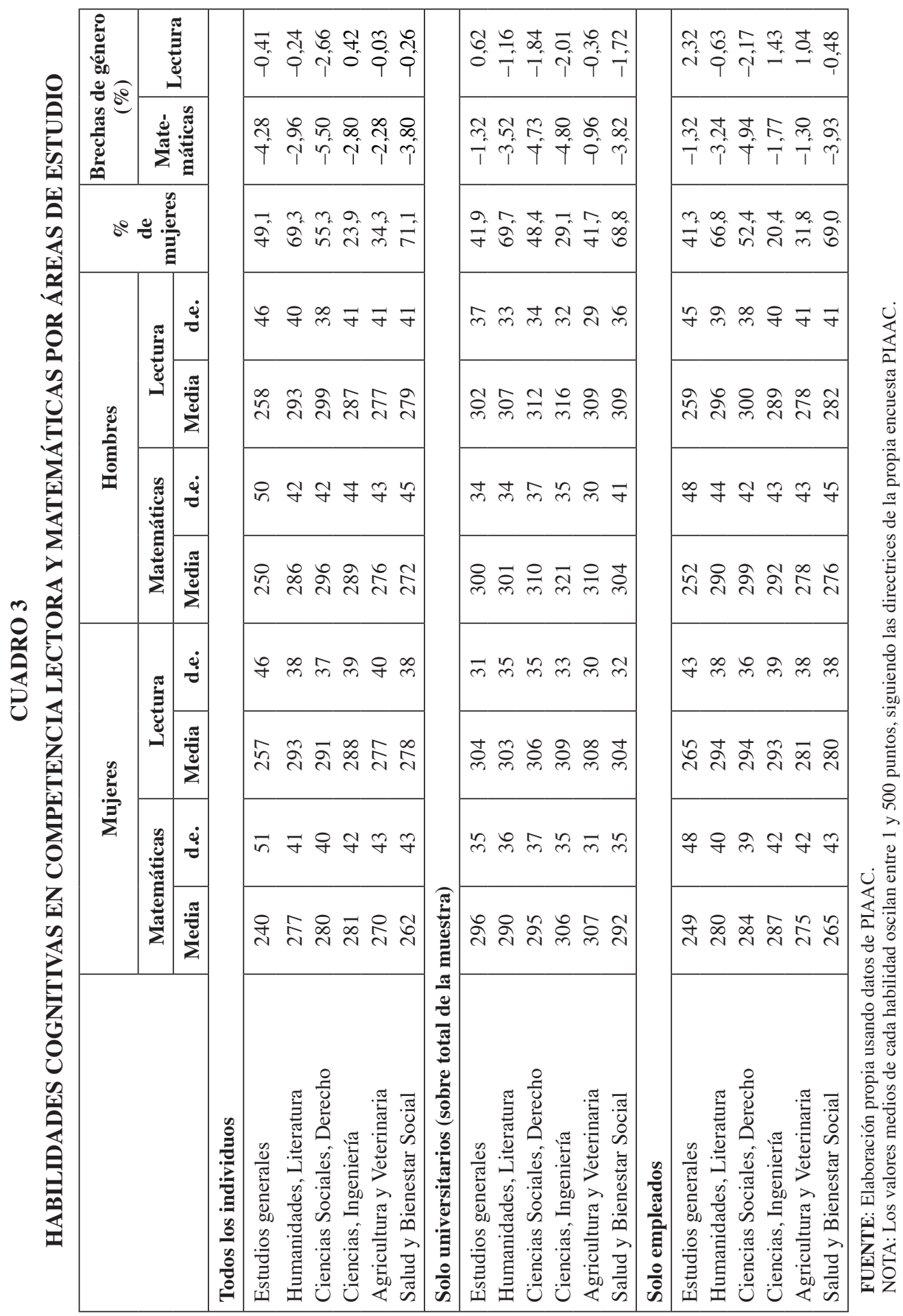




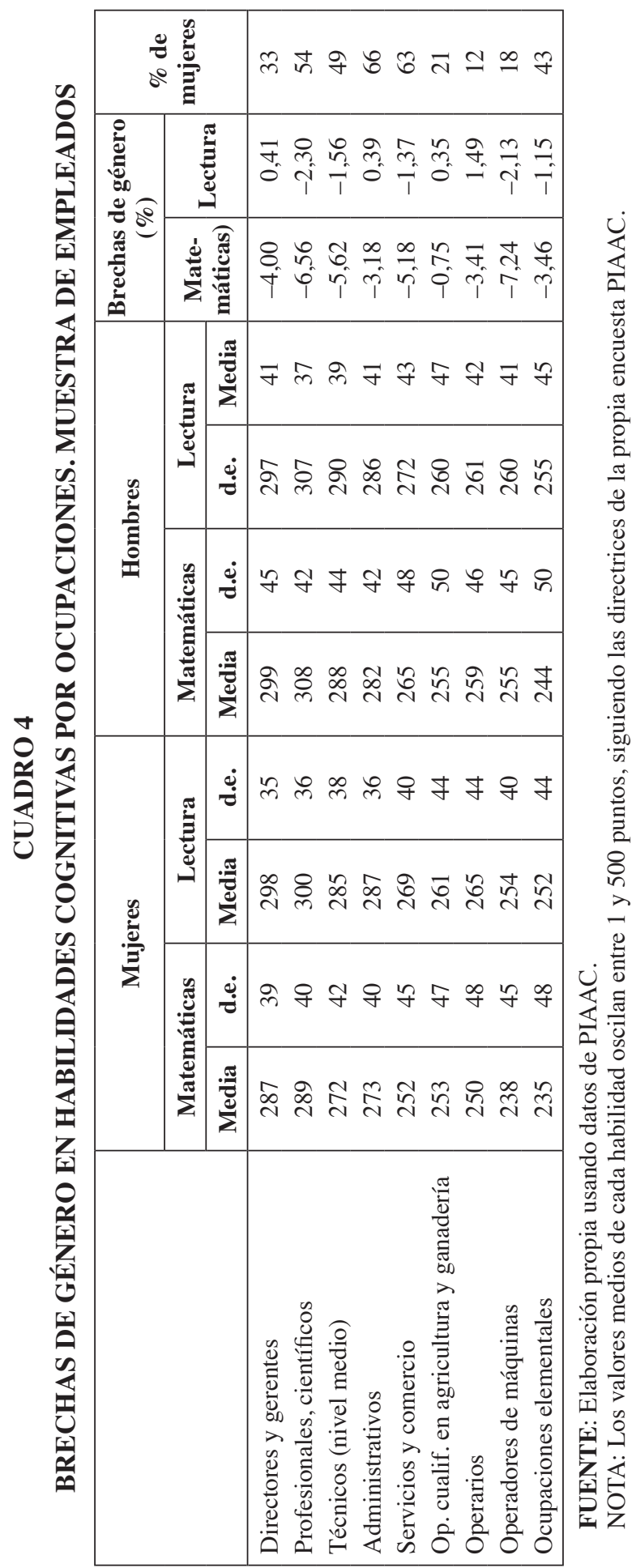


En primer lugar, la última columna del Cuadro 4 refleja un alto grado de feminización en algunas ocupaciones tales como el comercio o actividades administrativas. En segundo lugar, al igual que observamos en el área de estudio, estas ocupaciones feminizadas coinciden con ser ocupaciones donde los individuos muestran tener habilidades en matemáticas inferiores a la media.

\section{Análisis de las brechas de género en habilidades cognitivas: brecha sin ajustar frente a la brecha ajustada}

\subsection{Estrategia empírica}

En la sección anterior se ha presentado evidencia descriptiva que apunta la existencia de brechas de género en la competencia numérica independientemente de los grupos de edad, niveles educativos, áreas de estudio y tipos de ocupación. Por el contrario, en competencia lectora, las brechas son mínimas; por este motivo, en esta segunda parte del trabajo únicamente estimamos diferencias de género en competencias en matemáticas.

Los determinantes de estas brechas de género observada pueden ser muy variados. El objetivo de esta sección es estimar las brechas de género medias en habilidades numéricas a medida que se comparan individuos más similares, tanto en cuanto a sus características personales, como en las laborales. Estas brechas se denominan «ajustadas» o «condicionadas». La comparación entre las brechas de género mostradas anteriormente (observadas o no ajustadas) y las brechas que se muestran en esta sección (ajustadas) nos permite conocer hasta qué punto las diferencias de género tanto en características individuales como laborales (para el colectivo de trabajadores) ayudan a explicar las diferencias de género encontradas. En concreto, proponemos estimar un modelo econométrico con la siguiente estructura:

$$
\log \left(\text { Competencias }_{i}\right)=\alpha+\text { BMujer }_{i}+\sum_{k=1}^{K} \gamma_{k} X_{i k}+\sum_{j=1}^{J} \phi_{j} Z_{i j}+\sum_{m=1}^{M-1} \delta_{m}+\varepsilon_{i}
$$

Donde el índice $i$ hace referencia a los individuos y el término $d_{m}$ hace referencia al conjunto de $M-1$ variables ficticias de país $(M=31)$. La variable a explicar es el nivel de competencias cognitivas, más concretamente, la puntuación obtenida en los test de competencias en matemáticas. El objetivo fundamental es estimar el parámetro $b$, que nos mide la brecha de género en habilidades cognitivas condicionada a un conjunto de características observadas de los individuos que componen la muestra objeto de análisis. La variable mujer es una variable dicotómica que toma valor unitario si la observación se corresponde con una mujer y cero en caso contrario; dado que la variable a explicar la estimamos en logaritmos, este coeficiente nos permite expresar la brecha de género en términos porcentuales. El primer conjunto de regresores $-X_{i k}-$, hace referencia a las características individuales de los en- 
cuestados tales como edad, nivel de estudios, áreas de estudio y nivel educativo de los padres. La modelización de las características individuales relacionadas con las tres primeras variables se corresponde con la presentada en la sección de estadística descriptiva. El nivel educativo de los padres se modeliza con una variable ficticia que tomar valor la unidad si el padre o la madre tiene estudios universitarios y cero en caso contrario. Con este conjunto de variables podemos contrastar si las brechas de género observadas en las habilidades cognitivas se reducen o desaparecen una vez que condicionamos hombres y mujeres que exhiben similares niveles en estas características.

El segundo conjunto de regresores $-Z_{i-}$ - hace referencia a las características laborales del individuo entre las que se incluyen la experiencia laboral (en años trabajados), el grupo de ocupación (nueve categorías, tal y como se ha presentado en la sección de estadística descriptiva), el tipo de contrato (temporal/indefinido), las horas trabajadas (tiempo parcial/completo) y la titularidad de la empresa (privada/ pública). La introducción de estas variables nos permite contrastar si diferencias en las trayectorias laborales entre hombres y mujeres ayudan a explicar la brecha de género en las competencias cognitivas. Esta es una cuestión especialmente relevante para el estudio de brechas de género, puesto que las mujeres tienen, en media, trayectorias laborales menos estables que los hombres y con menor frecuencia se sitúan en ocupaciones que requieren niveles altos de cualificación. Todo ello podría repercutir en menores competencias cognitivas, fundamentalmente matemáticas, de las mujeres con respecto a los hombres, y consecuentemente mayor brecha de género en esta dimensión a lo largo del ciclo vital ${ }^{6}$. Esto solo tiene sentido, obviamente, cuando tratamos con el colectivo de personas ocupadas. Por este motivo, el modelo presentado se estimará para la muestra total de individuos y para la submuestra de trabajadores.

\subsection{Comportamiento de la brecha en competencias ajustadas por características individuales y laborales de los individuos: resultados}

El Cuadro 5A presenta los resultados de las brechas de género en matemáticas para todos los individuos a medida que se controla por diferencias individuales observadas entre hombres y mujeres. En la primera columna, como punto de referencia, presentamos las brechas de género sin ajustar, donde únicamente se incluye en la regresión los efectos fijos de país y la variable dicotómica mujer. En las siguientes columnas se computan sucesivas brechas de género en competencias en matemáticas a medida que comparamos hombres y mujeres más similares en cuanto a características individuales, como edad y nivel educativo, que reflejan de algún modo el capital humano adquirido y que por tanto esperamos que afecten al resultado en competencias en matemáticas.

${ }^{6}$ En DE LA RICA y REBOLLO (2018) se encuentra que las mayores habilidades cognitivas en matemáticas aumentan en 6-7 puntos porcentuales la probabilidad de estar empleado entre los entrantes al mercado de trabajo. 
Los resultados de la columna (1) muestran que la brecha en competencias numéricas es $-4,8$ por 100 , es decir, los hombres presentan unos resultados en competencias en matemáticas que son un 4,8 por 100 superiores a los de las mujeres en media, al comparar todos los hombres y todas las mujeres de un mismo país. En la columna (2) se añade información de los individuos relativa a la edad y el nivel de estudios y se obtiene que todos los coeficientes son estadísticamente significativos y tienen el signo esperado. En concreto encontramos que el nivel educativo, más que la edad, es una variable fundamental para entender la heterogeneidad observada en las habilidades en matemáticas. En concreto, el tener estudios secundarios se traduce en una puntuación en matemáticas un 20 por 100 superior, si se tiene estudios superiores no universitarios dicho efecto asciende al 28 por 100 , y al 38 por 100 si se tienen estudios universitarios, frente a tener solo estudios primarios. Obviamente, de estos resultados no podemos concluir una relación de causalidad entre nivel de estudios adquiridos y habilidades cognitivas en matemáticas, puesto que estos pueden estar motivados por un proceso de selección positiva (es decir, son los individuos con mayores habilidades numéricas los que con mayor probabilidad alcanzan niveles de estudios superiores). Los resultados relativos a la edad también muestran lo esperado, es decir, que las habilidades cognitivas en matemáticas se reducen a lo largo del ciclo vital, aunque su importancia es claramente inferior a la encontrada para los niveles educativos ${ }^{7}$. Sin embargo, a pesar de que la edad y la educación importan para entender los resultados en los test. de matemáticas, estas características no ayudan a reducir la brecha de género -ajustada, sino todo lo contrario, ya que esta crece hasta el $-5,2$ por 100 . Esto nos revela que las brechas de género que hemos encontrado anteriormente en las habilidades numéricas no pueden atribuirse a diferencias en los niveles educativos entre los hombres y las mujeres. De hecho, lo que esta estimación revela es que la brecha anteriormente observada no es más alta debido a que las mujeres han alcanzado, en media, niveles educativos superiores a los hombres. Si no hubiera sido así, la brecha media observada hubiera sido mayor.

Los resultados de la columna (3) muestran que el nivel educativo de los padres también influye en los resultados de los test en matemáticas aunque su importancia es también menor que el nivel educativo del individuo ${ }^{8}$. Adicionalmente, su contribución para explicar la brecha de género es pequeña puesto que esta solo se reduce levemente del 5,26 al 5,17 por 100. Finalmente, los resultados de la columna (4), que incluyen las áreas de estudio elegidas por los individuos como regresores, muestran que el tipo de área de estudios seleccionada influye en los resultados en los

\footnotetext{
${ }^{7}$ En concreto, encontramos que los jóvenes entre 24-29 años tienen puntuaciones en matemáticas 3,1 por 100 superiores a los mayores de 40 años. Para los individuos con edades entre 30-39 este diferencial cae al 2,2 por 100 .

8 Así, individuos con padres y/o madres con niveles de estudios superiores tienen scores que son un 3 a 4 por 100 superiores a los que no tienen padres/madres con dicho nivel de estudios. No obstante, esto de nuevo no se puede interpretar desde un punto de vista causal, ya que es de esperar que el nivel educativo de los padres esté altamente correlacionado con el nivel educativo de los hijos.
} 
CUADRO 5A

BRECHAS DE GÉNERO EN MATEMÁTICAS CONDICIONADAS POR CARACTERÍSTICAS INDIVIDUALES

(Todos los individuos)

\begin{tabular}{|c|c|c|c|c|}
\hline Variables & (1) & (2) & (3) & (4) \\
\hline Mujer & $\begin{array}{c}-0,0481 * * * \\
(0,00)\end{array}$ & $\begin{array}{c}-0,0526 * * * \\
(0,00)\end{array}$ & $\begin{array}{c}-0,0517 * * * \\
(0,00)\end{array}$ & $\begin{array}{c}-0,0415^{* * *} \\
(0,00)\end{array}$ \\
\hline Secundaria & & $\begin{array}{c}0,203 * * * \\
(0,01)\end{array}$ & $\begin{array}{c}0,204 * * * \\
(0,01)\end{array}$ & $\begin{array}{c}0,181 * * * \\
(0,01)\end{array}$ \\
\hline Estudios superiores no universitarios & & $\begin{array}{c}0,287 * * * \\
(0,01)\end{array}$ & $\begin{array}{c}0,283 * * * \\
(0,01)\end{array}$ & $\begin{array}{c}0,238 * * * \\
(0,01)\end{array}$ \\
\hline Estudios universitarios & & $\begin{array}{c}0,382 * * * \\
(0,01)\end{array}$ & $\begin{array}{c}0,364 * * * \\
(0,01)\end{array}$ & $\begin{array}{c}0,309 * * * \\
(0,01)\end{array}$ \\
\hline Edad: 24-29 (ref. 40-49) & & $\begin{array}{c}0,0311 * * * \\
(0,00)\end{array}$ & $\begin{array}{c}0,0216 * * * \\
(0,00)\end{array}$ & $\begin{array}{c}0,0213 * * * \\
(0,00)\end{array}$ \\
\hline Edad: 30-39 & & $\begin{array}{c}0,0225^{* * *} \\
(0,00)\end{array}$ & $\begin{array}{l}0,0194 * * * \\
(0,00)\end{array}$ & $\begin{array}{c}0,0182^{* * * *} \\
(0,00)\end{array}$ \\
\hline Estudios universitarios madre & & & $\begin{array}{c}0,0341 * * * \\
(0,00)\end{array}$ & $\begin{array}{c}0,0343^{* * * *} \\
(0,00)\end{array}$ \\
\hline Estudios universitarios padre & & & $\begin{array}{c}0,0411 * * * \\
(0,00)\end{array}$ & $\begin{array}{c}0,0406^{* * * *} \\
(0,00)\end{array}$ \\
\hline Humanidades, Literatura & & & & $\begin{array}{c}0,0460 * * * \\
\quad(0,00)\end{array}$ \\
\hline Ciencias Sociales & & & & $\begin{array}{c}0,0685^{* * * *} \\
(0,00)\end{array}$ \\
\hline Ciencias, Ingeniería & & & & $\begin{array}{c}0,0802 * * * \\
(0,02)\end{array}$ \\
\hline Agricultura y Veterinaria & & & & $\begin{array}{c}0,0379 * * * \\
(0,01)\end{array}$ \\
\hline Salud y Bienestar Social & & & & $\begin{array}{c}0,0195 * * * \\
(0,00)\end{array}$ \\
\hline Constante & $\begin{array}{c}5,668 * * * \\
(0,00)\end{array}$ & $\begin{array}{c}5,418 * * * \\
(0,01)\end{array}$ & $\begin{array}{c}5,414 * * * \\
(0,01)\end{array}$ & $\begin{array}{c}5,392 * * * \\
(0,01)\end{array}$ \\
\hline Observaciones & 123.160 & 107.619 & 107.619 & 107.619 \\
\hline $\mathrm{R}^{2}$ & 0,158 & 0,352 & 0,364 & 0,382 \\
\hline
\end{tabular}

FUENTE: Elaboración propia usando Datos PIAAC.

NOTA: Errores estándar robustos entre paréntesis. $* * * p<0,01 * * p<0,05 * p<0,1$. Todos los modelos incluyen efectos fijos de país. 
test en matemáticas ${ }^{9}$. Como era de esperar, los que eligen ciencias sociales y ciencias puras o ingeniería tiene mejores puntuaciones en esta área. En este caso, la brecha de género en matemáticas si se reduce en un punto porcentual, al 4,1 por 100. Por tanto, parece desprenderse que una parte de la brecha de género en competencias numéricas se debe, no a diferencias en el nivel educativo alcanzado, que como acabamos de ver de hecho ayuda a reducir la brecha de género, sino al área de estudio elegida. Las mujeres tienden a escoger áreas de estudio menos analíticas, a pesar de que el nivel de educación que alcanzan no es menor, y estas diferencias sí parecen explicar una parte de esas menores competencias numéricas que las mujeres muestran.

El Cuadro 5B presenta las estimaciones de la brecha de género ajustadas en competencias numéricas para el colectivo de trabajadores con el objeto de evaluar si estas se reducen en la población trabajadora cuando se comparan a individuos con similares características individuales y laborales ${ }^{10}$. En la columna (1) se presenta la brecha de género para la submuestra de trabajadores condicionando solo por el país de residencia del individuo. En la columna (2) se añaden las características individuales. De la estimación de estos dos modelos se aprende que la brecha en matemáticas -ajustada- es algo menor entre los individuos trabajadores con características individuales similares, en el entorno del 3,7 por 100. En la columna (3) se incluyen indicadores de ocupación usando como referencia «ocupaciones elementales». Los coeficientes estimados son positivos y estadísticamente significativos e indican que cuanto mayor sea la cualificación requerida en el puesto de trabajo mayores son las habilidades cognitivas en matemáticas de los individuos. Sin embargo, la brecha de género en matemáticas no se reduce al comparar hombres y mujeres que trabajan en la misma ocupación; por el contrario, aumenta levemente hasta el $-4,2$ por 100. Por tanto, a diferencia de la segregación por áreas de estudio, no parece que la segregación ocupacional por género pueda ser un factor que ayude a entender la brecha de género en competencias numéricas, una vez que se ha controlado por las características individuales. Finalmente, en la columna (4) se incluye un conjunto de regresores que ayudan a caracterizar mejor el tipo de empleo, como son el tipo de contrato, el tipo de empresa y la trayectoria laboral del individuo a través de su experiencia laboral medida en años. En general, estos nuevos regresores tienen un peso bastante menor para explicar el comportamiento de los test en matemáticas y apenas afectan a la estimación de la brecha de género, que se estima en un -3,9 por 100 .

La conclusión de esta sección, en consecuencia, es que la brecha de género en competencias numéricas es aproximadamente, el 5 por 100 para todos los individuos y el 4 por 100 para el colectivo de trabajadores y que ni las diferencias en capital humano ni las diferencias en la situación laboral de hombres y mujeres ayudan a explicar esa brecha.

${ }^{9}$ Nótese que en el caso de que los individuos no tengan estudios superiores y no indiquen el área de estudio, se asume que el área de estudio es un programa general. El área de estudios del programa general se incluye en la constante.

${ }_{10}$ Todos los modelos incluyen las características individuales de los individuos, pero dado que los resultados apenas cambian respecto a los mostrados en el Cuadro 5A, hemos optado por no mostrarlos para centrar el debate en el peso de las características laborales una vez controlado por las individuales. 
CUADRO 5B

BRECHAS DE GÉNERO EN MATEMÁTICAS OBSERVADA Y AJUSTADA POR VARIOS FACTORES

(Submuestra de empleados)

\begin{tabular}{|c|c|c|c|c|}
\hline & (1) & (2) & (3) & (4) \\
\hline Mujer & $\begin{array}{c}-0,0373^{* * *} \\
(0,00)\end{array}$ & $\begin{array}{c}-0,0372 * * * \\
(0,00)\end{array}$ & $\begin{array}{l}-0,0421 * * * \\
(0,00)\end{array}$ & $\begin{array}{c}-0,0399 * * * \\
(0,00)\end{array}$ \\
\hline Experiencia laboral (logs) & & & & $\begin{array}{c}0,0268 * * * \\
(0,00)\end{array}$ \\
\hline Directores y gerentes & & & $\begin{array}{c}0,114 * * * \\
(0,01)\end{array}$ & $\begin{array}{c}0,109 * * * \\
(0,01)\end{array}$ \\
\hline Profesionales, científicos & & & $\begin{array}{c}0,101 * * * \\
(0,01)\end{array}$ & $\begin{array}{c}0,0976 * * * \\
(0,01)\end{array}$ \\
\hline Técnicos y profesionales de nivel medio & & & $\begin{array}{c}0,0975^{* * * *} \\
(0,00)\end{array}$ & $\begin{array}{c}0,0968 * * * \\
(0,00)\end{array}$ \\
\hline Administrativos & & & $\begin{array}{c}0,0755^{* * * *} \\
(0,00)\end{array}$ & $\begin{array}{c}0,0726 * * * \\
(0,00)\end{array}$ \\
\hline Servicios y comercio & & & $\begin{array}{c}0,0729 * * * \\
(0,00)\end{array}$ & $\begin{array}{c}0,0712^{* * *} \\
(0,00)\end{array}$ \\
\hline Operadores de máquinas & & & $\begin{array}{c}0,0357 * * * \\
(0,00)\end{array}$ & $\begin{array}{c}0,0366^{* * *} * \\
(0,00)\end{array}$ \\
\hline Agricultores y ganadería & & & $\begin{array}{c}0,00837 \\
(0,01)\end{array}$ & $\begin{array}{c}0,00854 \\
(0,01)\end{array}$ \\
\hline Operarios & & & $\begin{array}{l}0,0316^{* * * *} \\
(0,01)\end{array}$ & $\begin{array}{l}0,0291 * * * \\
(0,01)\end{array}$ \\
\hline Constante & $\begin{array}{c}5,664 * * * \\
(0,00)\end{array}$ & $\begin{array}{c}5,427 * * * \\
(0,01)\end{array}$ & $\begin{array}{c}5,388 * * * \\
(0,01) \\
\end{array}$ & $\begin{array}{c}5,305^{* * *} \\
(0,01)\end{array}$ \\
\hline Nivel educativo & & $\mathrm{X}$ & $\mathrm{X}$ & $\mathrm{X}$ \\
\hline Edad & & $\mathrm{X}$ & $\mathrm{X}$ & $\mathrm{X}$ \\
\hline Área de estudio & & $\mathrm{X}$ & $\mathrm{X}$ & $\mathrm{X}$ \\
\hline Nivel educativo de los padres & & $\mathrm{X}$ & $\mathrm{X}$ & $\mathrm{X}$ \\
\hline Otras características laborales & & & & $\mathrm{X}$ \\
\hline Observaciones & 94.845 & 88.621 & 85.855 & 85.012 \\
\hline $\mathrm{R}^{2}$ & 0,129 & 0,350 & 0,371 & 0,374 \\
\hline
\end{tabular}

FUENTE: Elaboración propia usando con datos PIAAC.

NOTA: Errores estándar robustos entre paréntesis. $* * * p<0.01 * * p<0.05 * p<0.1$. Todos los modelos incluyen efectos fijos de país. Dentro del conjunto de regresores incluidos en otras características laborales se encuentran contrato temporal, tiempo-parcial, empresa grande (>200 trabajadores), empresa privada. En la constante se incluye el nivel de ocupación de menor cualificación. 


\section{Diferencias de género en competencias numéricas: disparidades entre países y posibles factores culturales}

El análisis realizado nos lleva a concluir que para entender los mecanismos que generan la brecha de género en competencias numéricas es preciso acudir a otros factores diferentes a los usuales que aproximan tanto el capital humano de los individuos como las condiciones laborales. Nótese que las brechas de género en la competencia numérica para el grupo de jóvenes son importantes y para este colectivo las condiciones laborales aún no deben ser un factor determinante para dichas brechas, como así apuntan nuestros resultados. La literatura empírica más reciente dedicada al estudio de las brechas de género coincide en señalar que los factores culturales parecen ser un mecanismo importante para entender las diferencias de género en competencias cognitivas en las economías desarrolladas (González de San Román y De la Rica, 2012; Nollenberger et al., 2016). Sin embargo, la base de datos PIAAC carece de indicadores de dichos factores culturales. Por tanto, en esta sección realizaremos un análisis exploratorio haciendo uso de otras fuentes de información. En concreto, tratamos de aprovechar la heterogeneidad de los países integrados en PIAAC para relacionar las brechas de género ajustadas por características individuales y laborales para cada país, obtenidas con los datos de PIAAC, con indicadores aproximados del calado social y cultural hacia la igualdad de género, provenientes del Índice global de la brecha de género, para los países incluidos en PIAAC.

Comenzamos mostrando las brechas de género observadas en competencias numéricas y lectoras en los diferentes países de la muestra en el Cuadro 6, mientras que en el Gráfico 1 se presentan las brechas de género en matemáticas ajustadas por características individuales y laborales para cada país. Dicha brecha ajustada se ha computado separadamente para cada país siguiendo la especificación del Cuadro $5 \mathrm{~B}^{11}$ y representa la brecha de género por países, que queda por explicar una vez que hemos condicionado por diferencias entre hombres y mujeres debidas a estas características.

Como primer elemento a destacar del Cuadro 6, cabe decir que existe una desventaja significativa de las mujeres relativa a los hombres en competencias matemáticas en absolutamente todos los países de la muestra y que la disparidad en la magnitud de la brecha es enorme. Países como Israel o Chipre muestran desventajas de la mujer frente al hombre del 10 y 9 por 100 , respectivamente, frente a países como Suecia, Polonia y Eslovaquia que exhiben las menores diferencias de género en competencias matemáticas, siendo la desventaja media de la mujer frente al hombre en torno al 1 por 100 .

11 Es decir, para obtener la brecha de género ajustada para cada país, estimamos el modelo de la columna (4) del Cuadro 5B para cada país. 


\section{CUADRO 6}

HABILIDADES COGNITIVAS EN COMPETENCIA LECTORA Y MATEMÁTICAS POR PAÍSES

(Todos los individuos)

\begin{tabular}{|c|c|c|c|c|c|c|c|c|c|c|}
\hline \multirow[b]{3}{*}{ Países } & \multicolumn{4}{|c|}{ Mujeres } & \multicolumn{4}{|c|}{ Hombres } & \multicolumn{2}{|c|}{$\begin{array}{l}\text { Brechas de } \\
\text { género }(\%)\end{array}$} \\
\hline & \multicolumn{2}{|c|}{ Matemáticas } & \multicolumn{2}{|c|}{ Lectura } & \multicolumn{2}{|c|}{ Matemáticas } & \multicolumn{2}{|c|}{ Lectura } & \multirow{2}{*}{$\begin{array}{c}\text { Mate- } \\
\text { máticas }\end{array}$} & \multirow{2}{*}{$\begin{array}{l}\text { Lec- } \\
\text { tura }\end{array}$} \\
\hline & Media & d.e. & Media & d.e. & Media & d.e. & Media & d.e. & & \\
\hline Israel & 215 & 51 & 227 & 39 & 237 & 44 & 235 & 37 & $-10,25$ & $-3,57$ \\
\hline Chipre & 208 & 52 & 226 & 47 & 226 & 53 & 232 & 48 & $-9,00$ & $-2,91$ \\
\hline Irlanda & 255 & 46 & 271 & 40 & 268 & 50 & 275 & 45 & $-5,41$ & $-1,39$ \\
\hline Chile & 252 & 48 & 277 & 41 & 265 & 53 & 278 & 45 & $-5,19$ & $-0,24$ \\
\hline Noruega & 283 & 41 & 289 & 36 & 298 & 45 & 293 & 39 & $-5,09$ & $-1,20$ \\
\hline Holanda & 286 & 40 & 296 & 38 & 300 & 42 & 300 & 39 & $-5,03$ & $-1,34$ \\
\hline Gran Bretaña & 262 & 47 & 278 & 42 & 275 & 52 & 280 & 47 & $-4,96$ & $-0,65$ \\
\hline Austria & 279 & 40 & 279 & 37 & 293 & 41 & 282 & 37 & $-4,96$ & $-1,33$ \\
\hline Bélgica & 285 & 40 & 286 & 38 & 299 & 43 & 289 & 40 & $-4,81$ & $-0,91$ \\
\hline Italia & 250 & 43 & 259 & 38 & 260 & 46 & 259 & 41 & $-4,14$ & $-0,03$ \\
\hline Singapur & 271 & 46 & 288 & 40 & 282 & 52 & 286 & 45 & $-4,13$ & 0,68 \\
\hline Japón & 287 & 37 & 303 & 32 & 299 & 39 & 306 & 32 & $-4,11$ & $-0,99$ \\
\hline Canadá & 271 & 45 & 285 & 41 & 282 & 49 & 284 & 44 & $-4,10$ & 0,38 \\
\hline Alemania & 278 & 45 & 281 & 41 & 289 & 47 & 282 & 43 & $-4,03$ & $-0,24$ \\
\hline España & 253 & 41 & 263 & 40 & 263 & 45 & 266 & 43 & $-3,88$ & $-0,98$ \\
\hline Francia & 263 & 47 & 275 & 40 & 272 & 49 & 274 & 43 & $-3,64$ & 0,48 \\
\hline Rep. Checa & 276 & 40 & 277 & 37 & 285 & 39 & 282 & 37 & $-3,39$ & $-1,51$ \\
\hline Dinamarca & 283 & 41 & 284 & 37 & 293 & 46 & 283 & 42 & $-3,38$ & 0,47 \\
\hline EEUU & 289 & 41 & 296 & 36 & 298 & 44 & 295 & 40 & $-3,32$ & 0,34 \\
\hline Eslovenia & 272 & 52 & 275 & 46 & 281 & 53 & 278 & 46 & $-3,20$ & $-1,05$ \\
\hline Finlandia & 291 & 40 & 307 & 37 & 300 & 44 & 301 & 41 & $-3,11$ & 1,78 \\
\hline Corea & 270 & 36 & 281 & 32 & 278 & 37 & 285 & 34 & $-2,87$ & $-1,44$ \\
\hline Lituania & 252 & 42 & 259 & 41 & 258 & 44 & 255 & 43 & $-2,35$ & 1,67 \\
\hline Estonia & 275 & 40 & 284 & 39 & 281 & 43 & 281 & 41 & $-2,25$ & 1,04 \\
\hline Turquía & 269 & 44 & 270 & 40 & 275 & 48 & 267 & 43 & $-1,94$ & 1,15 \\
\hline Grecia & 267 & 40 & 273 & 35 & 273 & 41 & 271 & 36 & $-1,93$ & 0,65 \\
\hline Nueva Zelanda & 272 & 43 & 273 & 38 & 276 & 45 & 271 & 39 & $-1,31$ & 0,94 \\
\hline Suecia & 278 & 44 & 277 & 37 & 281 & 45 & 277 & 37 & $-1,23$ & 0,00 \\
\hline Polonia & 265 & 43 & 277 & 41 & 268 & 48 & 271 & 45 & $-1,02$ & 2,31 \\
\hline Eslovaquia & 265 & 43 & 277 & 41 & 268 & 48 & 271 & 45 & $-1,02$ & 2,31 \\
\hline
\end{tabular}

FUENTE: Elaboración propia usando datos PIAAC.

NOTA: Los valores medios de cada habilidad oscilan entre 1 y 500 puntos, siguiendo las directrices de la propia encuesta PIAAC. 


\section{GRÁFICO 1 \\ BRECHAS DE GÉNERO EN MATEMÁTICAS AJUSTADAS POR CARACTERÍSTICAS INDIVIDUALES Y LABORALES POR PAÍSES}

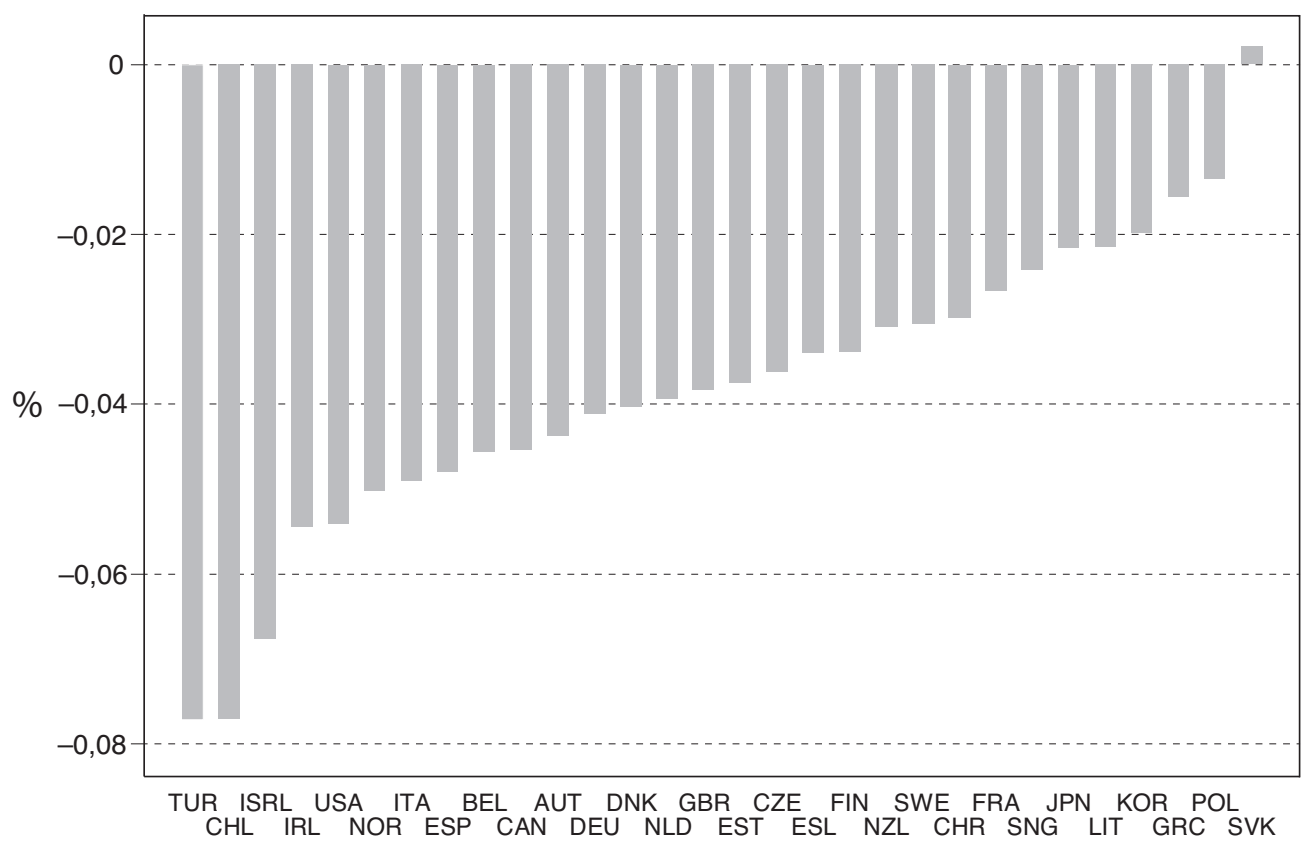

FUENTE: Elaboración propia usando datos de PIAAC.

Por otra parte, el Gráfico 1 confirma que las disparidades en la brecha de género observada por países se mantienen para la brecha de género ajustada por características individuales y laborales. En consecuencia, deben existir otros factores que generan la brecha de género en matemáticas más allá de características personales y laborales. A continuación trataremos de relacionar estas brechas ajustadas en matemáticas con indicadores de diferencias de género en estos países. Si fuera así, al menos exploratoriamente, estaríamos abriendo una posible línea de estudio a seguir para entender los determinantes de la brecha de género en matemáticas. Para ello, como indicador de la importancia cultural y social de la igualdad de género para cada país, tomamos el Índice de Igualdad de Género Global ${ }^{12}$ que proporciona anualmente el Foro Económico Mundial (World Economic Forum, The Global Gender Gap Report, 2012). Dicho índice contiene cuatro componentes, a saber, índice de igualdad en educación, en sanidad, en participación política y en oportunidades la-

${ }^{12}$ La brecha de género medida por el Índice Global, es la diferencia entre mujeres y hombres que se refleja en los logros o actitudes sociales, políticos, intelectuales, culturales o económicos. El Índice Global de Brecha de Género tiene como objetivo medir esta brecha en cuatro áreas clave: salud, educación, economía y política. Dado que el informe mide estas diferencias independientemente de los niveles generales de ingresos, algunos países relativamente pobres pueden tener un buen desempeño en el índice. 
borales. Dado que entre los países participantes en PIAAC la variabilidad en cuanto a los índices de igualdad en salud y educación es muy baja, se presenta únicamente la correlación entre el índice de igualdad de género global y el índice de igualdad en oportunidades laborales.

El Gráfico 2 presenta la correlación entre la brecha de género ajustada en competencias matemáticas y dichos índices de igualdad de género usando la variabilidad de los países. Si bien parece observarse una pequeña correlación entre las brechas de género en competencias numéricas y el índice de igualdad global, sí se observa una clara relación positiva significativa entre la brecha de género en competencias numéricas y la brecha de género en igualdad de oportunidades (correlación del 32 por 100). Esto revela que, a mayor desigualdad de oportunidades de las mujeres frente a los hombres, parece observarse mayor brecha de género en competencias numéricas

Este resultado indica que para entender los mecanismos que generan las brechas de género existentes en competencias numéricas a nivel internacional, sería necesario disponer de datos longitudinales y a nivel de país, tanto de variables que reflejaran valores culturales y/o normas sociales, como de competencias numéricas, de modo que se pudiera entender mejor la evolución de las diferencias de género en función de posibles cambios en los factores culturales y atribuir así posibles cambios

\section{GRÁFICO 2}

\section{BRECHAS DE GÉNERO EN HABILIDADES NUMÉRICAS AJUSTADAS CON RELACIÓN A LOS ÍNDICES DE IGUALDAD DE GÉNERO}
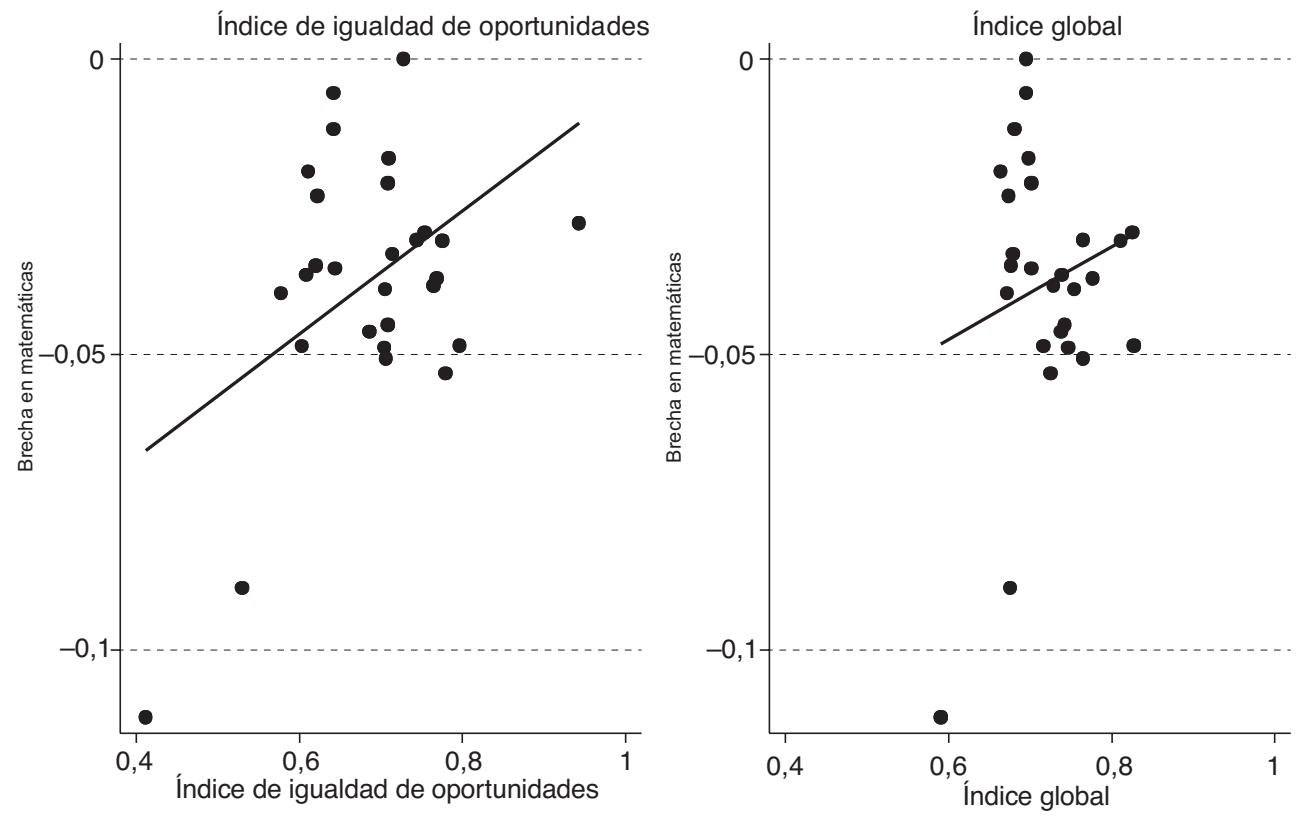

FUENTE: Elaboración propia usando datos PIAAC e Índice de Igualdad de Género Global que proporciona anualmente el Foro Económico Mundial. 
en las brechas en función de cambios culturales. Desafortunadamente, estos datos no existen en la actualidad, y todo lo que podemos hacer con este estudio en este sentido es simplemente mostrar una posible senda exploratoria por donde se podría, quizá, avanzar para entender los mecanismos que generan esas brechas de género en competencias numéricas.

Como ejercicio adicional, evaluamos las brechas de género en competencia matemática de la población adulta reflejada en PIAAC con brechas de género identificadas para la población joven aún en el proceso educativo tomando los datos de PISA-2012 $2^{13}$. Nótese que dichas brechas se pueden considerar ajenas a factores laborales y están más genuinamente relacionadas con factores sociales y culturales. En concreto, usando de nuevo la heterogeneidad por países, correlacionamos las brechas de género ajustadas de PIAAC con las brechas de género en matemáticas observadas derivadas de PISA. Adicionalmente, hacemos uso de la base de datos PISA para evaluar si entre los factores culturales que puedan estar detrás de la brecha de género en matemáticas resultan ser relevantes aquellos que hacen referencia a la actitud de los jóvenes hacia las matemáticas. Para medir dicha actitud nos centramos en dos variables: los nervios y la tensión que provocan las pruebas de matemáticas. Los datos de PISA muestran que los estudiantes que afrontan las pruebas de matemáticas con más nervios y tensión, obtienen peores resultados (PISA in Focus número 84).

El Gráfico 3 pone de manifiesto la existencia de importantes brechas de género en competencias en matemáticas, así como notables brechas de género en la actitud frente a las matemáticas, según los datos de PISA-2012. En el primer caso, las brechas son en general negativas para la mujer, de igual signo que las encontradas para la población adulta, mientras que en el segundo caso son positivas indicando que las chicas (15 años) se ponen más nerviosas y tensas ante las tareas y exámenes de matemáticas que los chicos de la misma edad ${ }^{14}$.

Finalmente, el Gráfico 4 se compone de tres figuras. El Gráfico del panel superior muestra la relación entre las brechas de género en matemáticas derivadas de PIAAC y las brechas de género en matemáticas derivadas de PISA. Encontramos una correlación positiva del 35 por 100. En el panel inferior se presenta la relación entre las brechas de género de PIAAC con las brechas de género en tensión -gráfico izquierdo- y nervios - gráfico derecho-, ante las actividades de matemáticas.

La conclusión exploratoria que se deriva de esta sección es que para entender las brechas de género en matemáticas es necesario acudir a edades mucho más tempranas que la edad adulta. Estas brechas se producen ya a la edad de 15 años, y parece existir un componente intangible, que es la ansiedad/nervios que la asignatura de matemáticas provoca, que afecta clara y negativamente a los resultados de las pruebas. Las niñas sufren significativamente mayor ansiedad/nervios que los niños, lo

${ }^{13}$ PISA es un test de competencias lectoras, matemáticas y ciencias que se realiza trianualmente a alumnos de 15-16 años de numerosos países desde 2003. Es una iniciativa de la OCDE.

${ }^{14}$ Esta brecha es la diferencia para cada país entre el porcentaje de mujeres que declaran que se ponen nerviosas/tensas cuando hacen ejercicios de matemáticas y el porcentaje de hombres que declaran que se ponen nerviosos/tensos cuando hacen ejercicios de matemáticas. 


\section{GRÁFICO 3 \\ BRECHAS DE GÉNERO EN COMPETENCIA MATEMÁTICA Y EN ACTITUD FRENTE A LAS MATEMÁTICAS SEGÚN PISA}
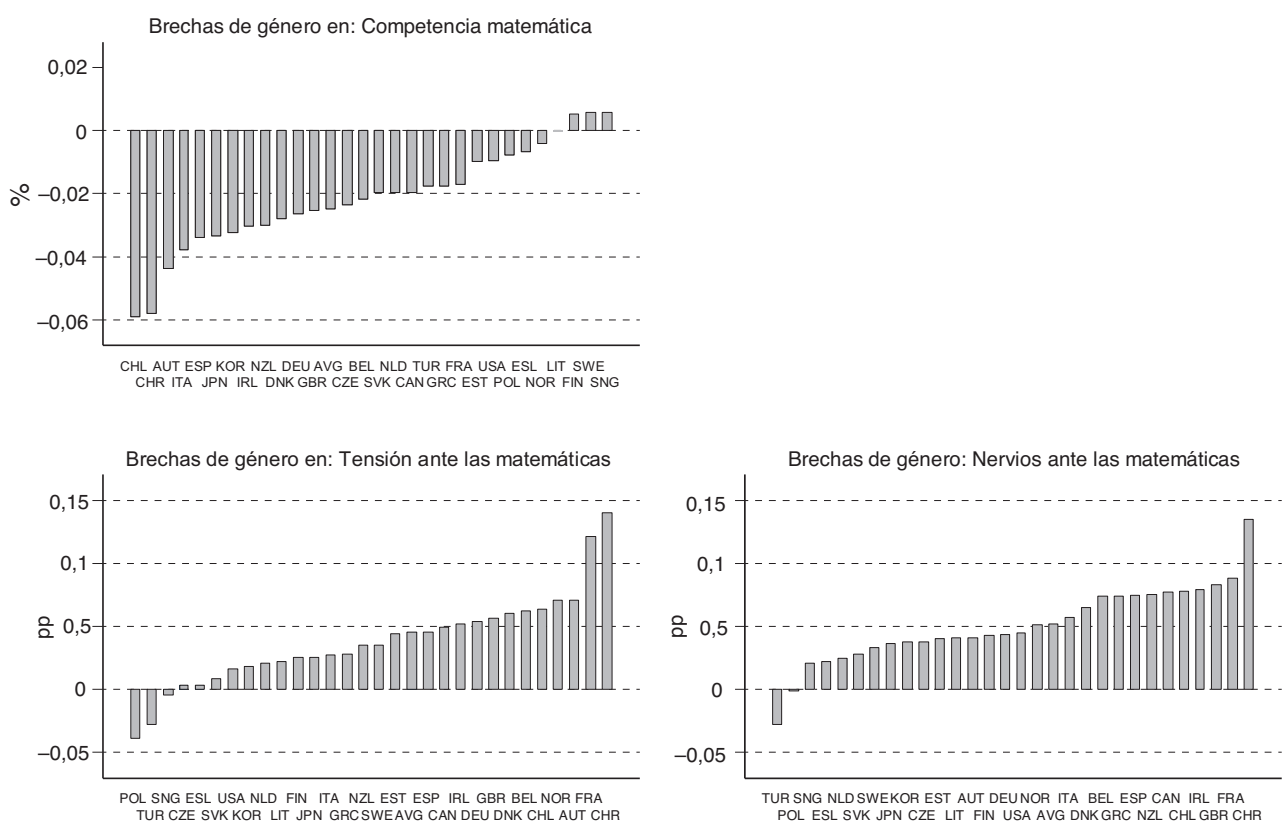

FUENTE: Elaboración propia usando datos de PISA.

que sin duda incita a pensar que este intangible podría estar detrás de la brecha de género en competencias matemáticas encontrada en este estudio. La falta de datos nos impide ir más allá de esta conclusión exploratoria, y sería necesario de disponer de datos longitudinales de estos intangibles para cada uno de los países, así como de pruebas en competencias lectoras, para poder justificar hasta qué punto esas diferencias en ansiedad/tensión están detrás de los hechos observados en este estudio, que apuntan a una brecha de género persistente en matemáticas de alrededor de un 4 por 100 en la población adulta. 


\section{GRÁFICO 4}

BRECHAS DE GÉNERO EN MATEMÁTICAS AJUSTADAS EN PIAAC FRENTE A DIFERENTES BRECHAS DE GÉNERO EN ACTITUDES FRENTE A LAS

\section{MATEMÁTICAS EN PISA}
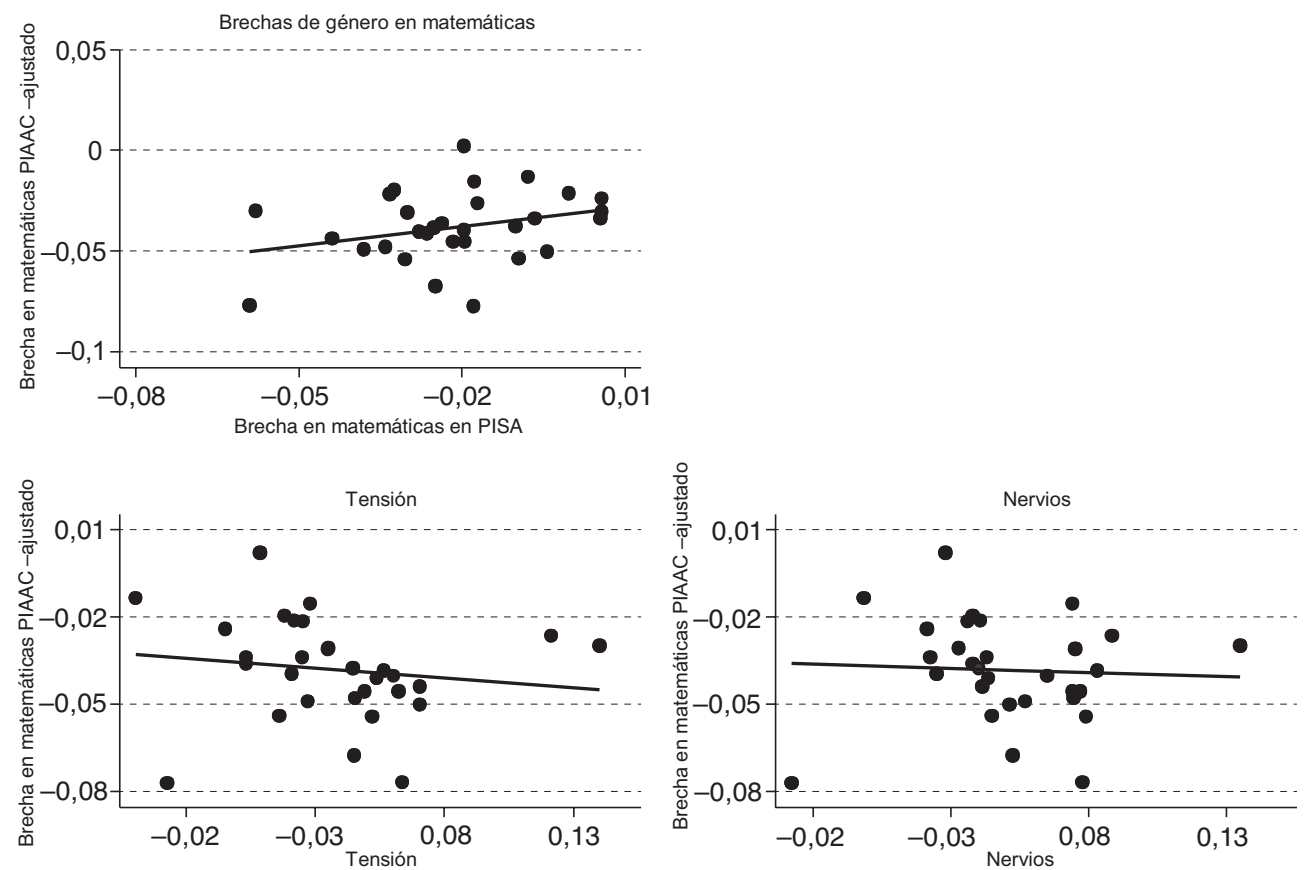

FUENTE: Elaboración propia usando datos de PIAAC y PISA.

\section{Conclusiones}

El papel de las políticas económicas ha sido y sigue siendo crucial para alcanzar un mayor nivel de igualdad en términos de género. Tradicionalmente, las políticas de género han ido dirigidas a reducir las brechas en características fácilmente observables, tales como los años de estudio como factor determinante de la trayectoria laboral futura, u otros como el fomento de la incorporación de la mujer al mercado de trabajo y a su permanencia en el mismo tras la maternidad. Sin embargo, siguen existiendo brechas de género en otras dimensiones más intangibles que finalmente se manifiestan en brechas de género en la población adulta y que se traducen en diferencias de oportunidades en el mercado de trabajo.

En este trabajo se ha mostrado una de estas dimensiones menos tangibles y se ha documentado la existencia de brechas de género en el ámbito de la competencia en matemáticas. El análisis se ha realizado condicionando hombres y mujeres a mostrar 
similares características individuales y laborales. El primer resultado importante de este trabajo es que incluso comparando a individuos similares en términos de características individuales y laborales, existe una brecha de género en la competencia en matemáticas que oscila entre el 3 y el 4 por 100 .

En segundo lugar y de forma tentativa, se trata de explorar por factores explicativos alternativos de dichas brechas. Conscientes de que los datos de PIAAC no nos permiten llegar a conclusiones determinantes, ofrecemos una serie de correlaciones entre las brechas de género ajustadas y variables que aproximan aspectos sociales y culturales hacia la igualdad de género. Encontramos que las brechas de género en matemáticas están positivamente correlacionadas con la «desigualdad» de oportunidades laborales. Aún más interesante, encontramos una relación positiva y sustancial entre las brechas de género mostradas en PIAAC y las que PISA 2012 arroja para los chicos y chicas de 15 años. Y además, estas brechas están fuertemente relacionadas con diferencias de género de un intangible como es la ansiedad/nervios frente a las matemáticas. Ese intangible afecta negativamente a los resultados en competencias matemáticas, y se observa que las niñas sufren mucha más ansiedad frente a las matemáticas que los niños. En consecuencia, creemos que se abre una línea de estudio muy interesante que desafortunadamente solo podrá ser desarrollada si se dispone de datos que permitan atribuir hasta qué punto estas diferencias de género en intangibles están detrás de las brechas de género en matemáticas. Por ahora, solo hemos podido documentar una conclusión exploratoria de este que puede ser el caso.

\section{Referencias bibliográficas}

[1] BLAU, F. D. y KAHN, L (2017). «The Gender Wage Gap: Extent, Trends and Explanations». Journal of Economic Literature, 55 (3), 789-865.

[2] DE LA RICA, S. y REBOLLO-SANZ, Y. F (2018). «From gender gaps in skills to gender gaps in wages: Evidence from PIAAC» (mimeo).

[3] GONZÁLEZ DE SAN ROMÁN, A. y DE LA RICA, S. (2012). «Gender Gaps in PISA Test Scores: The Impact of Social Norms and the Mother's Transmission of Role Attitudes». IZA Discussion Paper No. 6338.

[4] HANUSHEK, E. A.; SCHWERDT, G.; WIEDERHOLD, S. y WOESSMANN, L. (2015). «Returns to Skills Around the World: Evidence from PIAAC». European Economic Review, 73 (C), 103-130.

[5] HERTZOG, C.; KRAMER, A. F.; WILSOR, R. F. y LIDENBERGER, U. (2008). «Enrichment effects on adult cognitive development: can the functional capacity of older adults be preserved and enhanced?». Psychological Science in the Public Interest, 9, 1-65.

[6] KLEVEN, H. LANDAIS, C. y SOGAARD, J. E. (2019). «Children and Gender Inequality: Evidence from Denmark». NBER Working Paper, No. 24219.

[7] NOLLENBERGER, N.; RODRÍGUEZ-PLANAS, N. y SEVILLA, A. (2016). «The Math Gender Gap: The Role of Culture». American Economic Review, 106 (5), 257-61.

[8] OECD (2016). Skills Matter: Further Results from the Survey of Adult Skills. OECD Publishing, París. http://dx.doi.org/10.1787/9789264258051-en. 
[9] OECD (2018). Panorama de la educación 2016: Indicadores de la OCDE. Fundación Santillana, Madrid. https://doi.org/10.1787/eag-2016-es.

[10] OliveTti, C. y PETRONGOLO, B. (2016). «The Evolution of Gender Gaps in Industrialized Countries». Annual Review of Economics, 8 (1), 405-434.

[11] PISA (2012). PISA in Focus $N^{\circ} 88-H o w$ are school performance and school climate related to teachers' experience? PISA, OECD Publishing, París.

[12] PISA (2012). PISA 2012 Results: Ready to Learn: Students' Engagement, Drive and Self-Beliefs (Volume III), PISA, OECD Publishing, París.

[13] WORLD ECONOMIC FORUM (2012). The Global Gender Gap Report 2012. 